\section{Tiefe Hirnstimulation (DBS) beim idiopathischen Parkinson-Syndrom}

Wolfgang Fogel

Fachbereich Neurologie, DKD Wiesbaden

psychoneuro 2003; 29 (10): 454-456

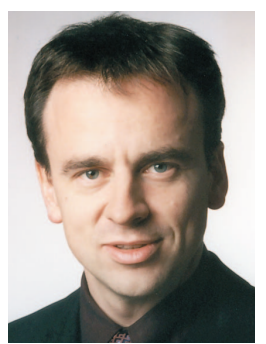

Wolfgang Fogel
Die tiefe Hirnstimulation (DBS) hat sich innerhalb des letzten Jahrzehnts als eine wichtige Behandlungsoption zur Behandlung des therapieresistenten Parkinson-Tremors sowie des fortgeschrittenen idiopathischen Parkinson-Syndroms mit Fluktuationen und Dyskinesien etabliert. Die Stimulation im Nucleus ventralis intermedius des Thalamus (Vim) führt zu einer deutlichen positiven Beeinflussung des Tremors ohne Besserung der anderen Kardinalsymptome. Durch die Stimulation im Nucleus subthalamicus (STN) oder Globus pallidus internus (Gpi) können alle Kardinalsymptome der Parkinson-Erkrankung gebessert werden. Darüber hinaus kommt es zu einer signifikanten Reduktion von Fluktuationen und Dyskinesien, die für die Patienten einen erheblichen Gewinn der Lebensqualität bedeutet. Das Verfahren ist sicher, allerdings besteht ein, wenn auch geringes, Risiko von intrazerebralen Blutungen und Infekten besonders bei älteren Patienten. Die Stimulations-induzierten Nebenwirkungen lassen sich in aller Regel durch eine Anpassung der Stimulationsparameter vermeiden. Insgesamt bietet das Verfahren eine hervorragende Ergänzung der Therapie des idiopathischen Parkinson-Syndroms bei entsprechend geeigneten (Dopa-responsiven) Patienten, die durch eine medikamentöse Therapie keine ausreichende Lebensqualität mehr erreichen.

$\mathrm{D}$ ie Einführung der tiefen Hirnstimulation bedeutet für die Behandlung des fortgeschrittenen idiopathischen ParkinsonSyndroms einen ähnlichen Fortschritt wie die Einführung von LDopa in den 60er-Jahren. Auch für die Behandlung verschiedener Dystonieformen, insbesondere generalisierter Dystonien, wird das Verfahren mit zunehmendem Erfolg eingesetzt. Das Verfahren wurde erstmals von Benabid (1) zur Behandlung des Parkinson-Tremors eingesetzt. Seitdem hat die tiefe Hirnstimulation, zumindest in den Industrienationen, zunehmend die älteren läsionellen Verfahren ersetzt.
Die ersten Stimulationselektroden wurden im Nucleus ventralis intermedius (Vim) innerhalb des Thalamus implantiert. Später kamen als weitere Zielgebiete, insbesondere zur Behandlung der fortgeschrittenen Parkinson-Erkrankung, der Nucleus subthalamicus (STN) und der Globus pallidus internus (Gpi) hinzu. Für die Behandlung von dystonen Bewegungsstörungen wird als Zielgebiet der Thalamus (Vim und Voa), zunehmend aber der Globus pallidus internus ( $\mathrm{Gpi}$ ) eingesetzt.

Mittlerweile sind, zumindest für die DBS bei der Parkinson-Erkrankung, ausreichende Daten zur Si- cherheit und Wirksamkeit des Verfahrens publiziert $(2,7)$. Diese Daten genügen zwar bislang noch nicht den strengen Kriterien der evidence based medicine (6), rechtfertigen aber den Einsatz bei Patienten, die medikamentös aufgrund von Nebenwirkungen wie Dyskinesien oder ausgeprägten Fluktuationen nicht ausreichend behandelt werden können (3).

\section{Prinzip der tiefen \\ Hirnstimulation}

Die Wirksamkeit der Stimulation mit hohen Frequenzen (130-200 Hz) wurde bereits im Rahmen der Läsionschirurgie entdeckt, als man am Zielpunkt vor Anbringen der Läsion mit einer Stimulationselektrode stimulierte und einen reversiblen Läsions-ähnlichen Effekt erzielte. Dank der technischen Entwicklung konnten später Dauerelektroden hergestellt werden, die mittels des Stereotaxieverfahrens durch ein Bohrloch zum jeweiligen Zielpunkt geführt und dort implantiert werden. An jeder Elektrode befinden sich vier einzeln ansteuerbare Kontakte durch die die Stimulationsenergie appliziert wird. Die Stimulationselektroden werden mit einem subklavikulär implantierten Stimulationsgenerator durch subkutan verlegte Ka- 
bel verbunden. Hierdurch wird eine chronische, 24-stündige Stimulation ermöglicht.

Das Wirkprinzip der Stimulation, bei der mittels eines kurzen (ca. 60-90 $\mu \mathrm{s}$ ) dauernden Impulses mit einer Frequenz von 130-180 Hz stimuliert wird, ist noch nicht geklärt. Wahrscheinlich kommt es durch die hohe Entladungsfrequenz zu einer Inhibition von Neuronen bzw. Axonen und dadurch zu einer funktionellen Ausschaltung der implantierten Kerngebiete. Diese Kerngebiete (z.B. der STN oder Gpi) sind bei der Parkinson-Erkrankung überaktiv und führen dadurch zur Zunahme der Parkinson-Kardinalsymptome wie Hypokinese/Akinese und Rigor. Durch die Ausschaltung dieser Kerngebiete durch Hochfrequenzstimulation kommt es zu einer eindrücklichen Besserung der ParkinsonSymptome.

\section{DBS bei der \\ Parkinson-Erkrankung}

Bei der Parkinson-Erkrankung werden drei Zielpunkte zur Elektrodenimplantation verwendet. Der Nucleus ventralis intermedius (Vim) innerhalb des Thalamus, der Nucleus subthalamicus (STN) und der Globus pallidus internus (Gpi).

Durch die Stimulation im Vim können sowohl der Parkinson-Tremor (alle Komponenten) als auch andere Tremorformen wie z.B. ein essenzieller Tremor oder ein zerebellärer Tremor bei der MS wirkungsvoll supprimiert werden. In einer randomisierten Studie konnte gezeigt werden, dass die Stimulation bei gleichem Effekt der Läsions-OP aufgrund geringerer Nebenwirkungen überlegen ist (5). Da die VimStimulation die anderen ParkinsonKardinalsymptome wie Rigor, Akinese und gestörte posturale Reflexe sowie die Spätkomplikationen wie Fluktuationen und Dyskinesien nicht beeinflusst, wird der Zielpunkt heutzutage nur noch bei Patienten mit ausgeprägtem, konservativ therapieresistenten Tremor ohne sonstige wesentliche Behinderung durch die Parkinson-Erkrankung gewählt.

Bei Patienten mit fortgeschrittenem idiopathischen Parkinson-Syn- drom mit therapieresistenten Fluktuationen und/oder Dyskinesien werden der STN und der Gpi als Zielpunkt gewählt. In beiden Zielpunkten werden sowohl die Akinese als auch der Rigor und der Tremor bei bilateraler Stimulation positiv beeinflusst. Bei der STN-Stimulation entspricht der Effekt in etwa dem Effekt, der durch die L-Dopa Gabe zu erreichen ist, bei der Gpi-Stimulation ist der Effekt etwas geringer ausgeprägt. Die Beeinflussung des Tremors durch die STN-Stimulation ist dem Therapieeffekt der Vim-Stimulation vergleichbar $(2,4)$. Auch hier zeigt sich eine etwas geringer ausgeprägte Besserung durch die Gpi-Stimulation. Durch die kontinuierliche 24-stündige Stimulation und den dadurch kontinuierlichen Effekt auf Tremor, Akinese und Rigor werden die Fluktuationen deutlich geglättet und Off-Phasen minimiert. Die Stimulation in beiden Zielpunkten führt außerdem zu einer deutlichen Reduktion von Dyskinesien. Bei der Gpi-Stimulation wird dies durch einen direkten anti-dyskinetischen Effekt der Stimulation erreicht. Eine Reduktion der dopaminergen Medikation ist hierbei allerdings nicht möglich. Bei der STN-Stimulation, durch die selbst ballistische Dyskinesien verstärkt bzw. ausgelöst werden können, kommt es trotzdem zu einer vergleichbaren, deutlichen Reduktion der Dyskinesien, da die dopaminerge Medikation aufgrund des guten Effektes der Stimulation auf die Off-Symptome um durchschnittlich 50\% reduziert werden kann. Welcher der beiden Zielpunkte vorzuziehen ist, ist derzeit noch nicht mit Sicherheit geklärt, da randomisierte Vergleichsstudien fehlen. Für die STN-Stimulation sprechen allerdings neben dem etwas besseren Effekt auf die Off-Symptome die Möglichkeit der Medikamentenreduktion sowie der im Vergleich zur Gpi-Stimulation niedrigere Stromverbrauch mit daraus resultierender längerer Batterielebensdauer (8). Für beide Stimulationsverfahren gilt, dass sie nur beim idiopathischen Parkinson-Syndrom mit erhaltener L-Dopa Antwort sinnvoll sind. Symptome, die nicht auf L-Dopa ansprechen, wie das On-Freezing oder auch nicht-responsive Sprachstörungen, sprechen auch auf die Stimulation nicht an. Entsprechend zeigt sich auch kein positiver Effekt bei anderen Parkinson-Syndromen wie der MSA, der CBD oder der progressiven supranukleären Paralyse.

\section{Risiken und Nebenwirkungen}

Wie bei jedem operativen Verfahren können auch bei der tiefen Hirnstimulation Komplikationen und Nebenwirkungen auftreten. Hierbei muss man zwischen irreversiblen und reversiblen Nebenwirkungen unterscheiden. Als gefürchtete Komplikation kann es bei einem geringen Teil der Patienten (2-3\%) zu einer intrazerebralen Blutung bedingt durch eine Gefäßverletzung durch das Einführen der Elektrode kommen. Oft handelt es sich hierbei um kleine Blutungen, die folgenlos bleiben. Selten können aber, besonders bei älteren Patienten mit ausgeprägter Hirnatrophie oder bei Patienten mit Arteriosklerose, auch folgenschwere Blutungen eintreten. Diese Risikogruppe sollte nach Möglichkeit von einer Elektrodenimplantation ausgeschlossen werden. Als zweite mögliche Komplikation kann es durch die Einbringung von Fremdmaterial zu Infektionen kommen. Hier ist dann in aller Regel eine Explantation der Stimulationselektroden mit der Möglichkeit einer Reimplantation nach mehrmonati-

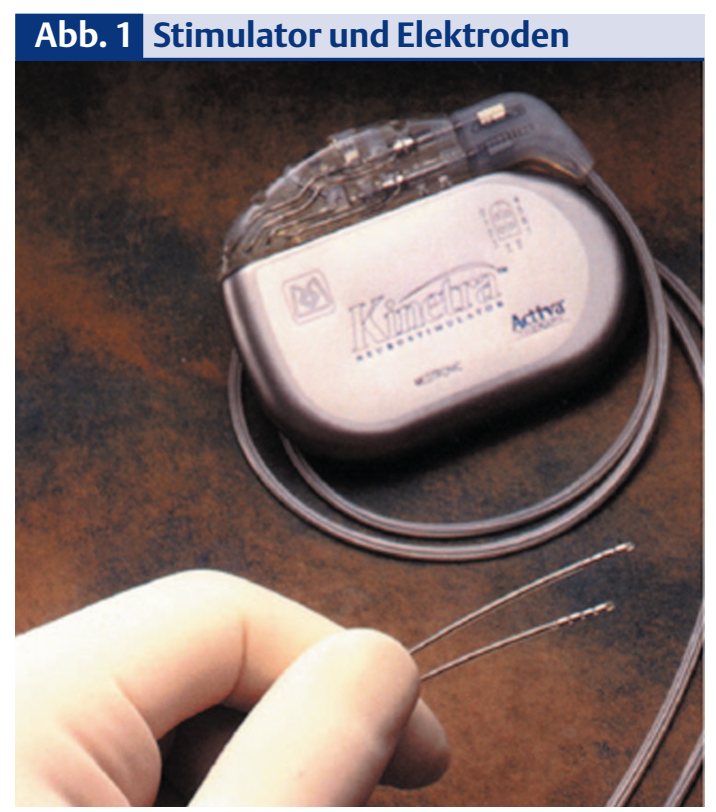


ger Wartezeit notwendig. Häufiger sind reversible Nebenwirkungen wie Parästhesien, Dysarthrie, Dyskinesien oder passagere Verwirrtheit, die in aller Regel durch eine Anpassung der Stimulationsintensität beseitigt werden können oder selbstlimitierend sind. Bezüglich der neuropsychologischen Nebenwirkungen der tiefen Hirnstimulation, besonders im STN, herrscht noch keine Klarheit. Es werden häufiger postoperative Zustände von Depressivität und Adynamie beobachtet, die sicher zum Teil auf die Reduktion der dopaminergen Medikation zurückzuführen sind. Allerdings wurden auch Stimulations-induzierte depressive Zustände bei unterhalb des STN im Bereich der substantia nigra gelegenem Elektrodenkontakt, aber auch maniforme Persönlichkeitsveränderungen bei Stimulation innerhalb des STN beobachtet. Diese Veränderungen werden auf die Beeinflussung der limbischen Ausflussbahnen der Basalganglien zurückgeführt. Diese neuropsychologischen Nebenwirkungen können durch eine Anpassung der Stimulationsparameter bzw. Adaption der dopaminergen Medikation in aller Regel gut kontrolliert werden. Insgesamt handelt es sich bei der tiefen Hirnstimulation um ein sicheres Verfahren, wenn die Ausschlusskriterien beachtet und die Einstellung der Stimulatoren von einem versierten Untersucher vorgenommen werden.

\section{Kosten}

Die unmittelbaren Kosten für die tiefe Hirnstimulation sind aufgrund der Material- und OP-Kosten hoch (Kosten von Stimulatoren und Elektroden ca. $20.000 €$.). Zu bedenken ist aber, dass - zumindest bei der STN-Stimulation - eine erhebliche Medikamenteneinsparung möglich ist und die indirekten Kosten (z.B. durch Krankenhausaufenthalte oder drohende Berentung) in vielen Fällen durch die wiedergewonnene Beweglichkeit und Unabhängigkeit der Patienten gesenkt werden. Eine große Deutschland-weite Studie, die auch die Lebensqualität der Patienten einbezieht, wird derzeit im Rahmen des MedNet Parkinson durchgeführt.

\section{Zukunft der DBS}

Auch wenn das Verfahren zum heutigen Zeitpunkt schon sehr ausgereift ist, bleiben noch offene Fragen und ungelöste Probleme. Verbesserungen im OP-Verfahren zur Minimierung der Risiken und Verbesserung der Zielpunktgenauigkeit sind wünschenswert. Eine Optimierung der bildgebenden Verfahren, z.B. mit MRT-kontrollierter Implantation der Elektroden, ist hier denkbar. Auch eine Verbesserung der Elektroden, um eine noch bessere Fokusierung der applizierten Stimulationsenergie $\mathrm{zu}$ erreichen, ist denkbar. Verbesserte Generatoren, die transkutan aufgeladen werden können, könnten die Behandlungskosten senken, da hierdurch ein Austausch der Batterien, der in aller Regel nach drei bis fünf Jahren notwendig ist, unnötig wäre. Ein besseres Verständnis der Wirkungsweise der Stimulation ist notwendig, um eine optimale Zielpunktdefinition zu ermöglichen bzw. die Modalität der applizierten elektrischen Impulse zu optimieren.

Ungeklärt ist darüber hinaus der optimale Zeitpunkt der Implantation im Krankheitsverlauf. Aufgrund des heutigen Kenntnisstandes wird die tiefe Hirnstimulation erst beim fortgeschrittenen, medikamentös ausbehandelten Parkinson-Syndrom eingesetzt. Es besteht allerdings ein hypothetischer neuroprotektiver Effekt der STN-Stimulation durch Verminderung der Glutamat-vermittelten Exzitation. Ein früherer Einsatz des Verfahrens wäre also denkbar. Daten hierzu fehlen aber derzeit und müssen durch größere, kontrollierte Studien erbracht werden.

\section{Summary}

During the last decade deep-brain stimulation (DBS) has been established as an important treatment option for the treatment of drugresistent Parkinson tremor as well as advanced idiopathic Parkinson's disease complicated by fluctuations and dyskinesias. Stimulation within the ventral intermedius nucleus of the thalamus (Vim) improves tremor without improving the other Parkinson symptoms. By stimulation of the subthalamic nucleus (STN) or the in- ternal pallidal globe (Gpi) all major motor symptoms of Parkinson's disease can be significantly ameliorated. Moreover, fluctuations and dyskinesias are significantly reduced leading to a marked improvement of the patient's quality of life. The method is safe, however there's a risk of intracranial bleedings or infection especially in elder patients. Stimulation-induced side effects can be avoided by adaption of the stimulation parameters. Altogether DBS is an outstanding addition for the therapy of patients with idiopathic (doparesponsive) Parkinson's disease who can not achieve a satisfactory quality of life with drug therapy alone.

Keywords: deep-brain stimulation Parkinson's disease - tremor - STN Gpi

\section{Literatur}

1. Benabid AL, Pollak P, Louveau A et al. Combined (thalamotomy and stimulation) stereotactic surgery of the VIM thalamic nucleus for bilateral Parkinson disease. Appl Neurophysiol 1987; 50: 344-346

2. Deep-Brain Stimulation for Parkinson's Disease Study Group: Deep-brain stimulation of the subthalamic nucleus or the pars interna of the globus pallidus in Parkinson's disease. N Engl J Med 2001; 345: 956-963

3. Deuschl G, Wenzelburger R, Kopper F et al. Deep brain stimulation of the subthalamic nucleus for Parkinson's disease: a therapy approaching evidence-based standards. J Neurol 2003; 250: 143-46

4. Krack P, Benazzouz A, Pollak P et al. Treatment of tremor in Parkinson's disease by subthalamic nucleus stimulation. Mov Disord 1998; 13: 907-914

5. Schuurman PR, Bosch DA, Bossuyt PM et al. A comparison of continuous thalamic stimulation and thalamotomy for suppression of severe tremor (see comments). N Engl J Med 2000; 342: 461-468

6. Task Force of the Movement Disorder Society. Management of Parkinson's Disease: an evidence-based review. Mov Disord 2002; 17: S1-S166

7. Tronnier VM, Fogel W, Krause $M$ et al. High frequency stimulation of the basal ganglia for the treatment of movement disorders: current status and clinical results. Minim Invasive Neurosurg 2002; 45: 91-96

8. Volkmann J, Allert N, Voges J et al. Safety and efficacy of pallidal or subthalamic nucleus stimulation in advanced PD. Neurology 2001; 56: 548-551

\section{Korrespondenzadresse:}

Dr. Wolfgang Fogel

Fachbereich Neurologie

Deutsche Klinik für Diagnostik

Aukammallee 33

65191 Wiesbaden 